\title{
Cryptogenic Liver Disease in Four Children: A Novel Congenital Disorder of Glycosylation
}

\author{
CLAUDIA MANDATO, LENA BRIVE, YOSHIAKI MIURA, JOSEPH ALEX DAVIS, NICOLINA DI COSMO, \\ STEFANIA LUCARIELLO, SEVERO PAGLIARDINI, NEUNG-SEON SEO, GIANCARLO PARENTI, RAFFAELLA VECCHIONE, \\ HUDSON H. FREEZE, AND PIETRO VAJRO
}

\begin{abstract}
Department of Pediatrics [C.M., NDC., S.L., G.P., P.V.], Department of Pathology [R.V.], University of Naples "Federico II", 80122 Naples, Italy; Glycobiology and Carbohydrate Chemistry Program [L.B., Y.M., J.A.D., N-S.S., H.H.F.], The Burnham Institute, La Jolla, CA 90237; Neonatal Screening Center [S.P.], A.O.O.I.R.M.-S. Anna, 10126 Turin, Italy
\end{abstract}

\begin{abstract}
We investigated the metabolic defect(s) of four children who presented with isolated cryptogenic chronic liver disease, coagulopathy, and abnormalities of several unrelated serum glycoproteins. Analysis of the patients' serum glycoproteins and fibroblasts suggest they have a novel congenital disorder of glycosylation (CDG). All had abnormal transferrin (Tf) isoelectric focusing (IEF) profiles. More detailed analysis of Tf by electrospray ionization mass spectrometry (ESI-MS) showed a plethora of abnormal glycosylations that included loss of 1-2 sialic acids and 1-2 galactose units, typical of Group II defects. Tf from two patients also lacked 1-2 entire oligosaccharide chains, typical of Group One disorders. Total serum N-glycans were analyzed by HPLC and matrix-assisted laser desorption/ionization mass spectrometry and also showed increased proportion of neutral glycan chains lacking sialic acids and galactose units. Analysis of patient fibroblasts eliminated CDG-Ia, through CDG-Ih, -I $L$ and CDG-IId. Our results suggest that a subset of children with clinically asymptomatic, cryptogenic hypertransaminasemia and/or liver steato-fibrosis may represent a novel type of CDG-X with an unknown defect(s). Clinicians are encouraged to test such patients for abnormal Tf glycosylation by ESI-MS. (Pediatr Res 59: 293-298, 2006)
\end{abstract}

$\mathrm{C}$ ongenital disorders of glycosylation (CDG) consist of at least 18 autosomal recessive metabolic disorders due to defects that alter protein $\mathrm{N}$-glycosylation (1). There are two groups. The first is defined by defects in the biosynthesis and transfer of a sugar chain from a lipid-linked oligosaccharide (LLO) precursor to newly synthesized proteins in the Endoplasmic Reticulum. Group One includes types Ia to $I L$. The second group is defined by defects in N-linked sugar chain processing in the Golgi, accounting for types IIa to IIf. Recently, defects in intracellular protein trafficking have been shown to cause a novel type of CDG that disrupts the biosynthesis of both $\mathrm{N}$ - and O-linked oligosaccharides (2). Thus, carbohydrate side chains of glycoproteins can be missing from the protein core (CDG I) or structurally altered (CDG II) (1, 3 ). Hypo- or misglycosylation is routinely determined by isoelectric focusing (IEF) of serum transferrin (Tf). Abnormal

Received February 16, 2005; accepted September 7, 2005.

Correspondence: Pietro Vajro, M.D., Department of Pediatrics, University of Naples "Federico II", Via Pansini 5 -80131 Naples, Italy; e-mail: vajro@unina.it

H.F. is supported by the March of Dimes Foundation and by National Institutes of Health Grant R01 DK55615.

DOI: 10.1203/01.pdr.0000196378.30165.26 glycosylation also can be assessed by electrospray-mass ionization mass spectrometry (ESI-MS) (4), matrix-assisted laser desorption/ionization mass spectrometry (MALDI) (5-7). Enzymatic assays or molecular analysis are used to identify the altered genes for both CDG types I and II (1). The clinical picture of CDG shows multi-systemic abnormalities. Severe CNS involvement is observed in practically all CDG patients, except CDG-Ib ( $\sim 20$ known patients) (1-3) ranging from mild developmental impairment to severe brain malformations and significant motor and cognitive dysfunction. One patient with CDG-Ih also had mainly hepato-intestinal problems (8), but others had severe developmental delay and multi-organ failure (9). Here we describe four children affected by novel forms of CDG-X presenting as isolated cryptogenic chronic liver disease, coagulopathy, and multiple serum protein abnormalities without other symptoms previously seen in known CDG patients.

\section{PATIENTS}

The series consists of four patients ( 2 sibs) who were born to healthy, unrelated parents. Clinical examination was normal in all but a slightly enlarged liver in the two sibs. None showed neurologic symptoms. Their initial presenting feature was hypertransaminasemia (Fig. 1) while other liver function tests were within normal limits. All the usual causes of liver disease [infectious, toxic, autoimmune and metabolic diseases, e.g., viral hepatitis B and $\mathrm{C}$, autoimmune hepatitis, coeliac disease, galactosemia and fructose intolerance, cystic fibrosis, $\alpha-1$ antitrypsin $(\alpha 1 \mathrm{AT})$ deficiency] were excluded.

Informed consent was obtained in all cases and the study was approved by the local Institutional Review Boards.

Cases one and two. Sibs, female Pt 1 and male Pt 2 had hypertransaminasemia since age two. It was discovered in $\mathrm{Pt}$ 1 before minor surgery, and in Pt 2 at a screening check-up. Both had increased cholesterol (x 1.5- 2 unv), creatine phos- 

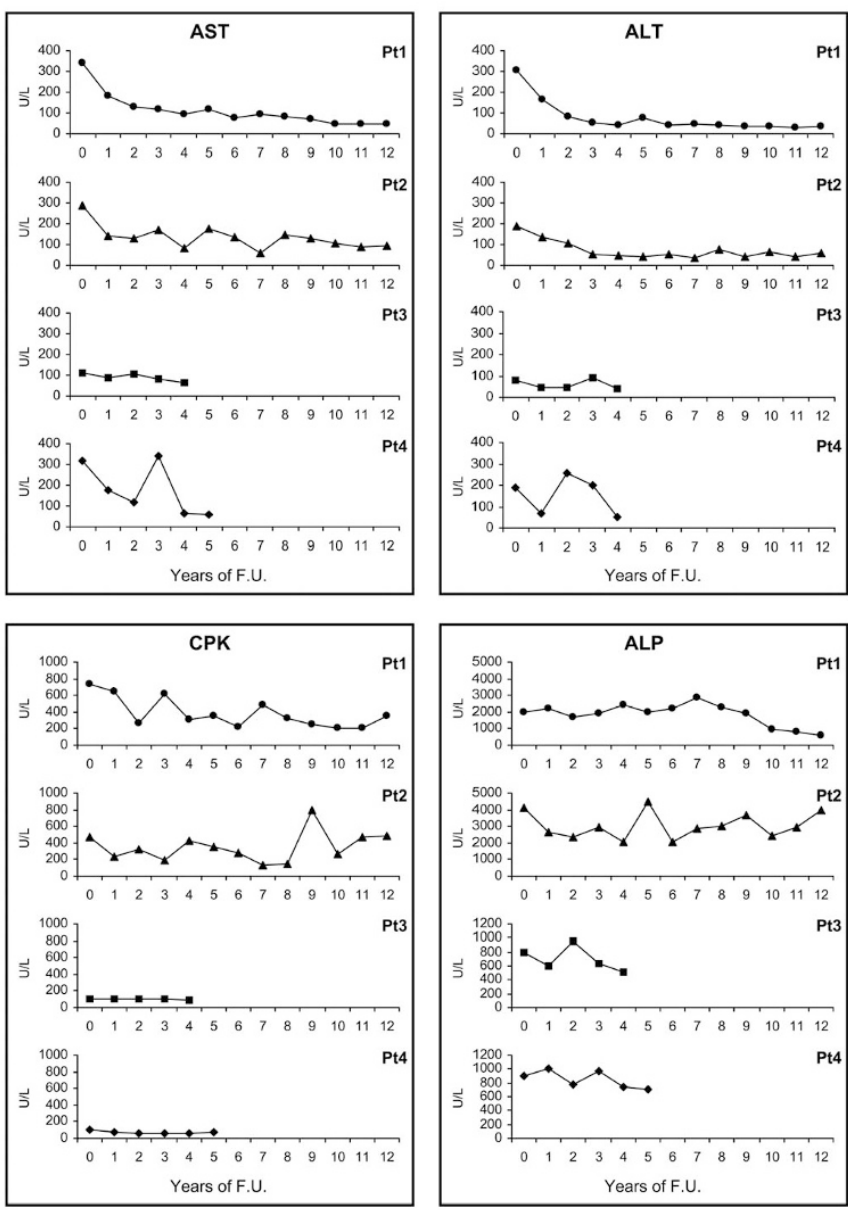

Figure 1. Serum levels of abnormal biochemical parameters [Alanine aminotransferase (ALT), aspartate aminotransferase (AST), creatine kinase (CPK), and alkaline phosphatase (ALP)] over time in the four patients.

phokinase and alkaline phosphatase (isoform pattern: bone $80 \%$; hepatic 5\%; intestinal $15 \%$ ) (Fig. 1). $\mathrm{Ca} / \mathrm{P}$ and serum $25 \mathrm{OH}$ vitamin $\mathrm{D}$ were normal. Because of reduced bone density based on computerized bone mineralometry a trial with 600.000 units of parenteral vitamin D was performed without effects on ALP values. Serum bilirubin and bile salts were normal. Coagulation parameters were normal or slightly reduced. Tf showed a persistent double peak. Haptoglobin was reduced in both $(0.2 \mathrm{~g} / \mathrm{L}$ and 0.4 , respectively; nv $0.5-3.1$ $\mathrm{g} / \mathrm{L})$. Ceruloplasmin $(6 \mathrm{mg} / \mathrm{dL}$ and $4 \mathrm{mg} / \mathrm{dL}$ respectively in pts 1 and 2; nv for age 20-46) and serum copper $(<40 \mathrm{mg} / \mathrm{dL}$ in both; nv 69-122) were very low in spite of a normal urinary excretion of copper at basal level (11 and $15 \mathrm{mg} / \mathrm{d})$ and after penicillamine administration (173 and $75 \mathrm{mg} / \mathrm{d}$ ).

Liver histology (Fig. 2A,B) showed mild periportal fibrosis and focal steatosis. Electron microscopy (courtesy of Prof. Sternlieb; NY, USA) showed no Wilsonian mithochondrial abnormalities.

Patients one and two liver copper content was 318 and 250 micrograms/g dry weight, respectively (normal values: $<50$ micrograms vs. Wilson disease or chronic cholestasis or newborn liver $>250$ micrograms); iron content was normal. Study of haplotypes for Wilson disease (linkage analysis for Wilson disease by polymorphic markers D13s295, D13s301,
D13s296, D13s298, localized inside and around Wilson disease gene) and ATP7B gene sequencing molecular analysis of DNA (courtesy of Dr G Loudianos, and Dr M. Pirastu, Cagliari, Italy) however were considered absolutely not consistent with a diagnosis of Wilson disease. Iron metabolism was normal. Nuclear magnetic resonance of brain and liver did not show increased metal storage. A liver biopsy, repeated three years later, confirmed the same findings in the two sibs. Their parents have normal liver function tests, serum cholesterol, CPK, ALP, and ceruloplasmin.

As shown in Figure 1, after a 12 y follow-up, transaminase levels are normal in $\mathrm{Pt} 1$, and tend toward lower values in $\mathrm{Pt}$ 2. Serum ALP, CPK, cholesterol, Tf, ceruloplasmin and copper are still abnormal. On the contrary basal and post penicillamine urinary copper levels remain normal. The multiple unrelated biochemical abnormalities, along with liver steatosis and mild fibrosis, prompted CDG testing.

Case three. Pt 3 had mild hypertransaminasemia discovered at age two during an episode of acute uricaria. Serum a1AT $(0.76 \mathrm{~g} / \mathrm{L} ; \mathrm{nv} 0.9-2.0 \mathrm{~g} / \mathrm{L})$ and ceruloplasmin $(17 \mathrm{mg} / \mathrm{dL} ; \mathrm{nv}$ $20-46 \mathrm{mg} / \mathrm{dL}$ ) were slightly below the normal values. However $\alpha 1 \mathrm{AT}$ deficiency and Wilson disease were ruled out by $\alpha 1 \mathrm{AT}$ IEF test and liver histology (see below), and serum, urinary and liver copper levels, respectively. PT (down to $55 \%$ ) and PTT (up to 50s) were occasionally abnormal. Multiple coagulation factors are consistently low without signs of disseminated intravascular coagulopathy (normal platelets and FDP), severe liver disease or cholestasis. Oral or intra muscular vitamin $\mathrm{K}$ administration had no effect. Anti nuclear antibodies showed a slight positivity (1:80), those against smooth muscle, mitochondrion, liver-kidney microsomes, and liver cytosol were negative. Persistent hypertransaminasemia prompted a liver biopsy at age six that showed microvacuolar steatosis and a mild portal inflammation. A short course of ursodeoxycholic acid and steroids did not modify the biochemical parameters. At age nine, a new liver biopsy confirmed mild portal fibrosis and focal steatosis but inflammation was no longer present (Fig. 2, panels $\mathrm{C} 1$ and $\mathrm{C} 2$ ). On the basis of liver steatosis, hypertransaminasemia, and coagulation defects, CDG was investigated.

Case four. Pt 4 had hypertransaminasemia revealed at age one during an episode of hypoglycemia and intermittent metabolic acidosis. Ultrasonography showed liver brightness. Liver biopsy revealed macro-microvesicular periportal steatosis (Fig. 2, panel D). In spite of mild liver dysfunction, he had coagulopathy (PT 50\%, PTT 44 s) with persistent decreased coagulation factors. $\mathrm{Tf}(1.55 \mathrm{~g} / \mathrm{L} ; \mathrm{nv} 2.3-4.5 \mathrm{~g} / \mathrm{L}), \alpha 1 \mathrm{AT}(1.2$ $\mathrm{g} / \mathrm{L}$; nv $1.4-2.4 \mathrm{~g} / \mathrm{L}$; slow allele $\mathrm{M}$ variant at IEF); haptoglobin $(0.3 \mathrm{~g} / \mathrm{L} ; \mathrm{nv} 0.5-3.1 \mathrm{~g} / \mathrm{L})$ and ceruloplasmin $(19 \mathrm{mg} / \mathrm{dL} ; \mathrm{nv}$ $20-46 \mathrm{mg} / \mathrm{dL}$ ) were also decreased. Wilson disease was ruled out by normal values of serum, urinary and liver copper levels. Fructose aldolase B and fructose 1-6 bisphosphatase deficiency in particular were ruled out by enzyme assays on liver biopsy specimen (courtesy of professor Gitzelman, Zurich, Switzerland). The combination of liver steatosis, coagulopathy and decreased levels of several serum glycoproteins, prompted CDG testing, which showed an abnormal Tf pattern. Because of a spontaneous improvement in blood glucose 


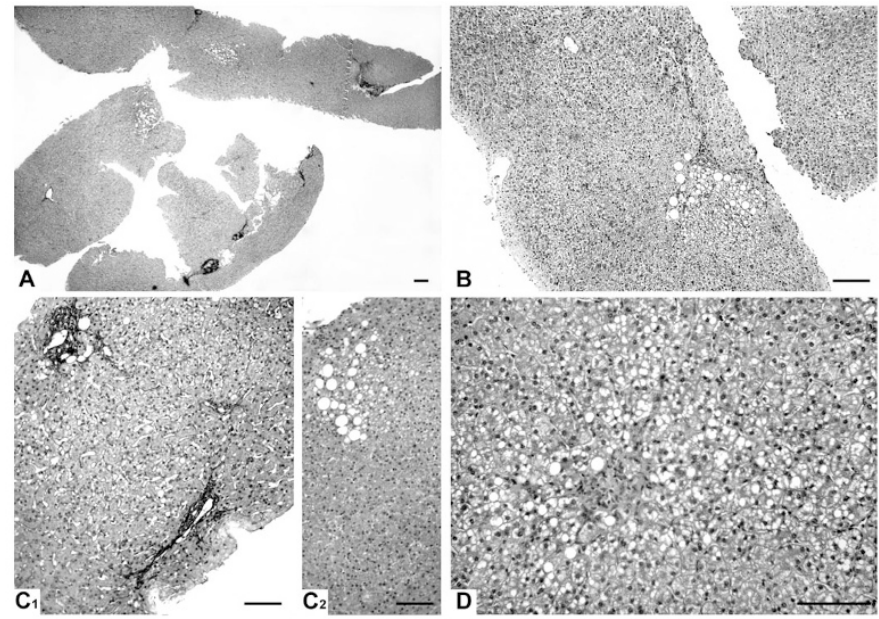

Figure 2. Histologic pictures of patients' needle (panels $A, B, C$ ) and surgical (panel $D$ ) liver biopsies. Panel $A$ (upper left). Red Sirius $40 \times$, patient Pt 1: mild portal fibrosis and focal steatosis. Panel B (upper right). EE $100 \times$, patient Pt 2: mild portal fibrosis with periportal steatosis Panel $C$ (lower left). EE 100×, patient Pt 3: mild portal fibrosis (C1) associated with focal steatosis visible in another field of the same specimen (C2) Panel $D$ (lower right). EE $200 \times$, patient Pt 4: macro-microvesicular periportal steatosis. Bars $=100 \mu \mathrm{m}$

levels and hypertransaminasemia, parents decided to stop further diagnostic tests.

\section{METHODS}

IEF analysis of serum Tf was previously described (10). Phosphomannomutase 2 (PMM2; EC 5.4.2.8) and phosphomannose isomerase (PMI; EC 5.3.1.8) assays and metabolic labeling of patient fibroblasts with $\left[2-{ }^{3} \mathrm{H}\right] \mathrm{man}-$ nose and LLO analysis by HPLC were described previously (11).

ESI-MS analysis of Tf. Procedure was adapted from (4). Tf was isolated from $100 \mu \mathrm{L}$ serum by binding to anti-Tf beads (Affigel 10, Bio-Rad, in 50 $\mathrm{mM}$ Tris- $\mathrm{HCl} 0.15 \mathrm{M} \mathrm{NaCl}, \mathrm{pH} 7.2$ ) and elution with $0.1 \mathrm{M}$ glycine/2\% acetic acid $\mathrm{pH} 2.5$ and neutralized by $1 \mathrm{M}$ Tris-base. The amples were bound to a C18-zip tip (Millipore, USA) rinsed by $5 \%$ acetic acid and eluted in 3-5 $\mu \mathrm{L}$ $5 \%$ acetic acid in methanol:acetonitrile:water (50:25:25). The isolated proteins $(1.5 \mu \mathrm{L})$ were analyzed by nanospray-MS using an API 3000 mass spectrometer (PE-SCIEX, Canada), operated in the positive ion mode at $700 \mathrm{~V}$.

Analysis of $\mathbf{N}$-glycans from serum. Serum $(10 \mu \mathrm{L})$ was denatured in $1 \%$ SDS and glycans released with PNGaseF and purified on graphitized carbon (12). A portion was fluorescently labeled at the reducing end with 2-aminobenzamide (2-AB) (13) and separated into neutral and anionic glycans by HPLC (14). Glycan profiles were determined from eight control sera and from a pool of 10 different sera. Duplicate analyses were highly reproducible, showing $<3 \%$ variation. The average glycan distribution \pm SEM was: neutral, $18.2+/-1.9 ; 1$ sialic acid, $19.9+/-1.9 ; 2$ sialic acids, $47.4+/-1.1 ; 3$ sialic acids, $11.8+/-1.4 ; 4$ sialic acids, $2.7+/-0.3$. Another portion of the released glycans was separated into neutral and anionic species on carbograph. After methyl esterification of sialic acids (15), the samples were analyzed by MALDI-TOF in the positive ion mode (16).

Presumptive structures were assigned to glycan masses based on established biosynthetic pathways and known structures previously identified in serum glycoproteins (5-7). Relative abundance of each species was calculated from peak area of the MALDI spectra adjusted for the proportion of neutral and anionic glycans in the HPLC pools.

Exclusion of hereditary fructose intolerance (HFI). In patients one, two and three, hereditary fructose intolerance was investigated by searching the aldolase B gene for the following mutations: MD $\Delta 4$, A149P, A174D, Y203X, L256P, N334 K by the polymerase chain reaction-amplification refractory mutation system, PCR-ARMS, this represents about $90 \%$ of HFI mutations in the Italian population, and mutation A337V (Mlu N1 restriction endonuclease digestion). Sequence analysis of the patients' entire HFI gene region encoding aldolase B vs. normal controls was also performed (courtesy of professor F. Salvatore and Dr F. Esposito, CEINGE Naples, Italy). In patient four, fructose aldolase B and 1,6 bisphosphatase deficiency were assayed in liver tissue.

\section{RESULTS}

Analysis of Tf glycosylation. Tf IEF patterns in all patients were abnormal (Fig. 3) and sialidase digestion (not shown) indicated misglycosylation. In Pt 1 and Pt 2, disialo-Tf and trisialo-Tf are increased; patients $\mathrm{Pt} 3$ and $\mathrm{Pt} 4$ show an increase of all the less sialylated forms. The patterns were compatible with Group II disorders, but the patients' clinical features were not.

To determine more precisely the nature of the Tf abnormality, it was immunopurified and analyzed by ESI-MS.4 Some molecules from the sibs lacked 1-2 sialic acids and 1 galactose residue. Pt 3 also lacked these sugars, and in addition, she also lacked 1-2 entire oligosaccharide chains, typical of Group One disorders (Fig. 4). Pt 4 also lacked 1-2 entire chains, but the loss of individual sialic acid residues was nearly insignificant. Patients typically fall into either group one or two, but not both.

Analysis of total serum N-glycans. To determine whether other liver-derived glycoproteins also have abnormal $\mathrm{N}$ glycans, the free sugar chains were released enzymatically and fluorescently labeled at the reducing end with 2-amino benzamide. HPLC separates sugar chains into molecules with 0,1 , 2, 3 or 4 sialic acids as shown in Fig. 5. Compared with normal serum, the patients showed significant increased proportion of neutral sugar chains and decreases in molecules with 2 sialic acids. The mass of each neutral or charged glycan pool was determined by MALDI-MS and likely structures assigned based on established biosynthetic pathways and previous analysis of serum-derived $\mathrm{N}$-glycans $(5,6)$. Figure 6 shows the distribution of some of the most prominent neutral species. All patients had significant increases in core fucosylated chains lacking 1-2 galactose residues. In normal sera, nonfucosylated glycans containing 1-2 terminal N-Acetylglucosamine units are undetectable, but are substantially enriched in all patients, especially in patient Pt 3, whose ESI-MS Tf analysis (Fig. 4 Peak N) also showed loss of an entire Nglycan, with the remaining glycan having 2 terminal $\mathrm{N}$ Acetylglucosamine residues. Although MALDI analyses cannot determine whether entire chains are absent, they do show that all the patients synthesize variable amounts of incomplete glycans lacking sialic and galactose residues. We cannot determine whether these variations represent different primary defects or individual variations of the same primary defect.

\section{CDG}

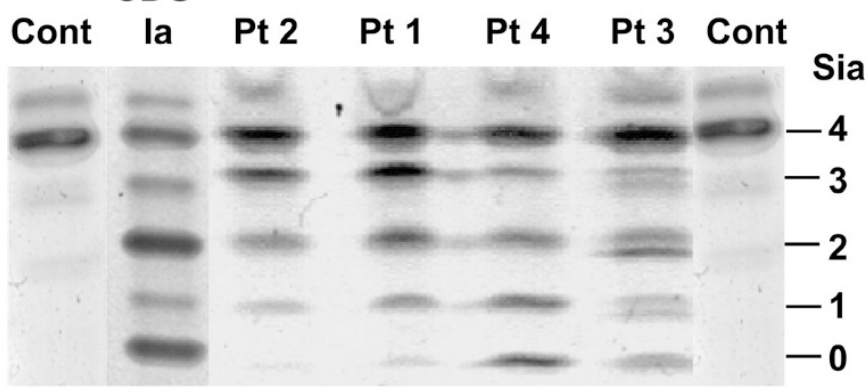

Figure 3. IEF of $\mathrm{Tf}$ from control and patients sera. Sera $(0.2 \mu \mathrm{L})$ from control (cont) and patients were subjected to IEF and the separated Tf bands were visualized by applying anti-Tf directly on the gel. The number of sialic acids (Sia) per molecule (1-4) is indicated on the right. 

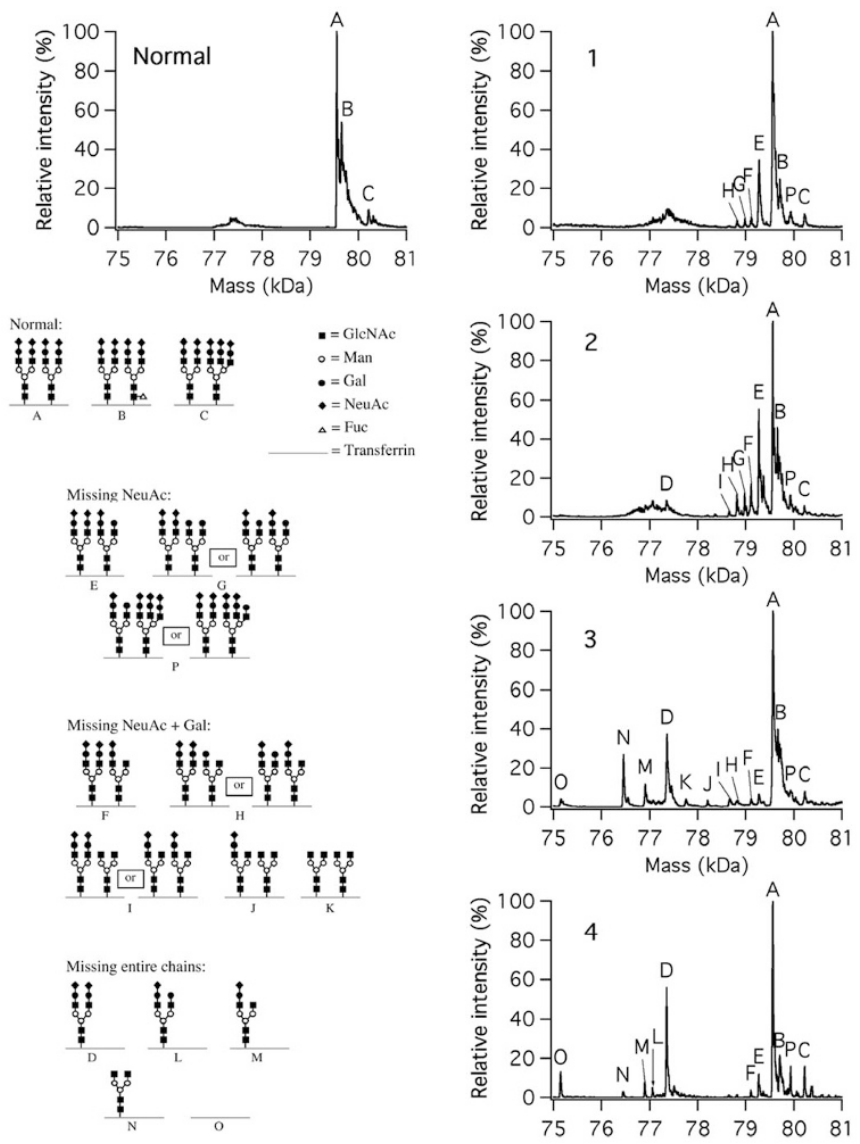

Figure 4. ESI-MS analysis of purified Tf. Tf was immunopurified from serum and immediately analyzed by ESI-MS. Normal and patients are indicated by the numbers in the upper left corner of each individual spectrum. Letters adjacent to the peaks indicate the predicted oligosaccharide structures shown at the left of the spectra and are based on the determined mass of the peaks. The key for symbols of the monosaccharides are shown just below the normal spectrum. GlcNAc, N-acetylglucosamine; Man, mannose; Gal, galactose; NeuAc, sialic acid and Fuc, fucose.

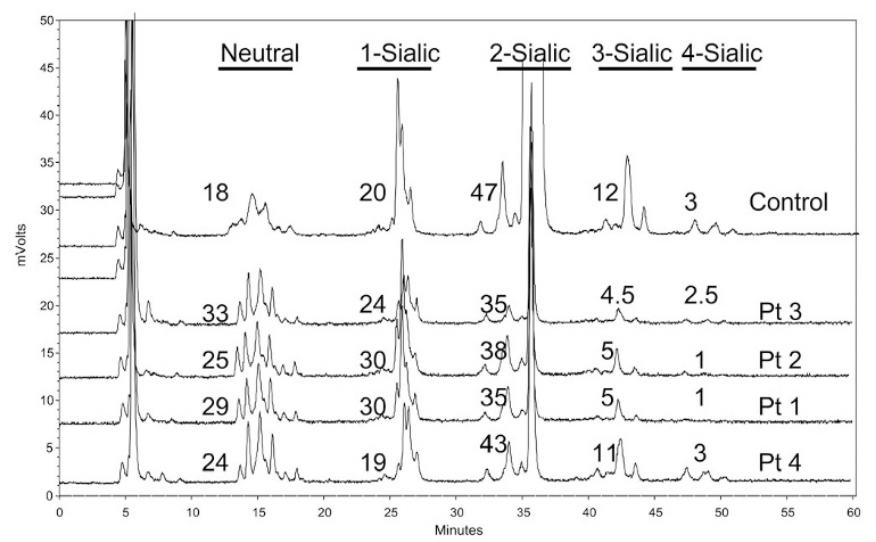

Figure 5. HPLC analysis of 2-aminobenzamide-labeled N-glycans from control and patient sera. $\mathrm{N}$-glycans were released from total serum of controls and indicated patients, labeled with 2-aminobenzamide and separated by HPLC into a series of neutral oligosaccharides and ones containing 1, 2, 3 and 4 sialic acids. Bars indicate the summed areas and numbers show the percentage of the total. See Methods for details.

Analysis of patients fibroblasts. Fibroblasts were available from Pt 1, Pt 2 and Pt 3. Their PMM and PMI activities were within the normal range, eliminating CDG-Ia and CDG-Ib.

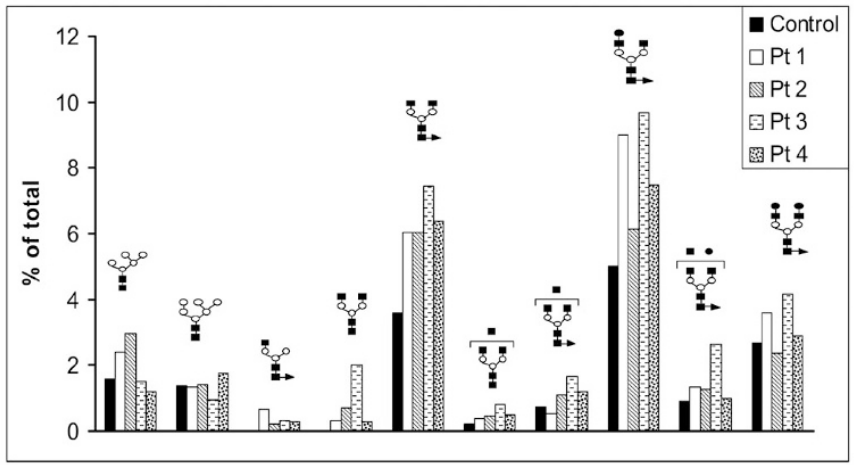

Figure 6. Distribution of Neutral N-glycans from control and patient sera. Neutral glycans were analyzed by MALDI spectrometry in positive ion mode, and each peak was assigned a structure based on mass. The ratio of each peak to total peak area in the spectra was calculated and adjusted for the percentage of neutral oligosaccharides shown in Figure 5. Monosaccharide symbols are the same as in Figure 4. Brackets between the glycan structure and monosaccharide symbol above it indicate that the specific location of the monosaccharide on the chain was not determined.

Metabolic labeling of these fibroblasts with $\left[2-{ }^{3} \mathrm{H}\right]$ Mannose and analysis of LLO showed normal size, thus eliminating CDG-Ic through CDG-Ih and CDG-IL. $\beta$-1,4 Galactosyltransferase (EC 2.4.1.90) activity in fibroblasts was also normal, eliminating CDG-IId. Hereditary fructose intolerance was ruled out in all patients and none of them had ever consumed alcohol.

\section{DISCUSSION}

The CDGs are a new group of inherited multisystem disorders. Coagulopathy is a consistent finding of both CDG groups I and II. Most have a prominent involvement of CNS and various dysmorphic features. CDG Ia is the most common type with about 400 known patients $(1,3)$ with a wide array of clinical features including lipodistrophy and cerebellar hypoplasia associated with various dysmorphisms, mental retardation, coagulopathy, impaired function of kidney, heart, and liver. The remarkable clinical variability has suggested that CDG in general probably remains largely underdiagnosed (17).

Novel forms of CDG (CDG-X) are steadily increasing $(5,6$, $18-20)$. Because scores of genes are used for the synthesis and function of glycoconjugates, undefined cases of CDG are likely to be identified by analysis of $\mathrm{N}$-glycosylation of plasma proteins.

Although several CDG-X clinical presentations have been reported, none of them is restricted to only liver involvement, sparing other organ systems (21-25). The four children described here had normal neurologic assessment and were evaluated because of a mild, chronic, cryptogenic hypertransaminasemia.

In all patients, several liver diseases were considered over the years. Investigation of CDG by Tf IEF analysis was triggered by the association of histologic steatosis and mild fibrosis as described in typical cases of CDG (26) with abnormalities of several unrelated serum glycoproteins including coagulation factors in pts 3 and 4 (27-30). Although it has 
been reported that primary liver dysfunctions may lead to possible secondary glycosylation defects, we believe that this is not the case of our series since the diagnostic work-up did rule out most etiologies of known liver diseases.

Known causes of interference with the IEF results, such as HFI and alcohol abuse, were ruled out by appropriate tests and clinical history as well.

In all patients the course of biochemical abnormalities was benign, with a general trend toward improvement of serum levels of liver enzymes and coagulation parameters, seemingly independent of medical treatments, as sometimes reported in CDG patients (1). Slightly decreased ceruloplasmin levels have been reported in a CDG patient (28) as in our pts 3 and 4 who had no other copper metabolism abnormalities. In contrast, the two sibs (pts 1 and 2) presented very low levels of serum ceruloplasmin as a persistent finding. Their consistently normal copper urinary levels, and molecular and haplotype studies for Wilson disease, and the favorable trend of liver function tests however are seemingly not related to typical Wilson disease. The increased liver copper content could be non specific as seen in children with a variety of non Wilsonian liver diseases (31). The possibility of a concomitant heterozygote status for a pathologic mutation causing Wilson disease unmasked by the hepatopatology caused by the CDG might still theoretically exist. Further studies in other patients are necessary to assess whether ceruloplasmin and copper abnormalities are characteristic of a novel CDG-X syndrome.

CDG must be taken into consideration in cases of increased serum CPK levels in patients with no evidence of muscular disease (18). Increased vitamin D-resistant serum levels of ALP with abnormal bone density found in two patients has also not been previously reported. However, hypoglycosylation of noncollagenous bone proteins has been suggested to contribute to the osteopenia found in rare instances of CDG I, which might be related to our findings (27).

Several methods were used to assess the patients' glycosylation. All had Tf IEF patterns typical of Group Two defects. ESI-MS analysis of Tf showed patterns consistent with Group Two defects in sibs pt 1 and pt 2, but pt 3 appeared to have ESI-MS patterns expected for a combination of both Group One and Group Two defects. Pt 4 had a pattern typical of Group One, with only a very slight decrease in sialylation. Other CDG-I patients have increased fucosylation and decreased branching of sugar chains in Tf (6) and total serum (32). Our results primarily show loss of both sialic and galactose from $\mathrm{Tf}$ and total serum $\mathrm{N}$-glycans. Biantennary fucosylated species containing 1-2 galactose residues were especially prominent compared with controls. It is noteworthy that a recent study described altered glycosylation of plasma proteins in patients with liver cirrhosis (33) showing increase in bisecting GlcNAc added by $N$-acetylglucosaminyltransferase III and progressive loss of galactose with progressive liver fibrosis.

A growing number of CDG-X patients lack both galactose and sialic acid on serum glycans $(5,6)$. Some also have defective O-linked glycosylation, indicated by analysis of Apo CIII (20,34); our patients also had abnormal ApoCIII (unpublished data). Mutations in proteins that traffic and organize the glycosyltransferases and nucleotide sugar transporters in the Golgi can also alter glycosylation (2), greatly expanding CDG candidate genes. The abnormal glycosylation seen in these patients would be compatible with defects that alter trafficking. Additional molecular studies will be needed to identify the primary defects in these children.

In conclusion, a subset of clinically asymptomatic, cryptogenic hypertransaminasemia and/or liver steato-fibrosis associated to abnormalities in serum proteins in children may represent one or more novel types of CDG. This novel presentation with liver disease and no neurologic involvement should alert physicians to check Tf glycosylation status, preferably using the more informative ESI-MS approach, rather than IEF.

Acknowledgments. We thank Dr. Erik Eklund for helpful discussions, analysis of MALDI spectra and construction of Fig. 6. Regarding pt 4 we thank Prof. Beat Steinmann for suspecting CDG, Dr. sc. nat. Nils U. Bosshard for the enzymatic exclusion of CDG Ia and Ib, and Prof. T. Hennet for the LLO analysis which was normal (Zurich, Switzerland).

\section{REFERENCES}

1. Marquardt T, Denecke J 2003 Congenital disorders of glycosylation: review of their molecular bases, clinical presentations and specific therapies. Eur J Pediatr 162:359_ 379

2. Wu X, Steet RA, Bohorov O, Bakker J, Newell J, Krieger M, Spaapen L, Kornfeld S, Freeze HH 2004 Mutation of the COG complex subunit gene COG7 causes a lethal congenital disorder. Nat Med 10:518-523

3. Freeze HH 2001 Congenital disorders of glycosylation and the pediatric liver. Semin Liver Dis 21:501-515

4. Lacey JM, Bergen HR, Magera MJ, Naylor S, O'Brien JF 2000 Rapid determination of transferrin isoforms by immunoaffinity liquid chromatography and electrospray mass spectrometyery. Clin Chem 47:513-518

5. Butler M, Quelhas D, Critchley AJ, Carchon H, Hebestreit HF, Hibbert RG, Vilarinho L, Teles E, Matthijs G, Schollen E, Argibay P, Harvey DJ, Dwek RA, Jaeken J, Rudd PM 2003 Detailed glycan analysis of serum glycoproteins of patients with congenital disorders of glycosylation indicates the specific defective glycan processing step and provides an insight into pathogenesis. Glycobiology 13:601-622

6. Mills P, Mills K, Clayton P, Johnson A, Whitehouse D, Winchester B 2001 Congenital disorders of glycosylation type I leads to altered processing of N-linked glycans, as well as underglycosylation. Biochem J 359:249-254

7. Mills PB, Mills K, Mian N, Winchester BG, Clayton PT 2003 Mass spectrometric analysis of glycans in elucidating the pathogenesis of CDG type IIx. J Inherit Metab Dis 26:119-134

8. Chantret I, Dancourt J, Dupre T, Delenda C, Bucher S, Vuillaumier-Barrot S, Ogier De Baulny H, Peletan C, Danos O, Seta N, Durand G, Oriol R, Codogno P, Moore SE 2003 A deficiency in dolichyl-P-glucose : Glc1Man9GlcNAc2-PP-dolichyl alpha 3-glucosyltransferase defines a new subtype of congenital disorders of glycosylation. J Biol Chem 278:9962-9971

9. Schollen E, Frank CG, Keldermans L, Reyntjens R, Grubenmann CE, Clayton PT, Winchester BG, Smeitink J, Wevers RA, Aebi M, Hennet T, Matthijs G 2004 Clinical and molecular features of three patients with congenital disorders of glycosylation type Ih (CDG-Ih) (ALG8 deficiency). J Med Genet 41:550-556

10. Westphal V, Enns GM, McCracken MF, Freeze HH 2001 Functional analysis of novel mutations in a congenital disorder of glycosylation Ia patient with mixed Asian ancestry. Mol Genet Metab 73:71-76

11. Westphal V, Murch S, Kim S, Srikrishna G, Winchester B, Day R, Freeze HH 2000 Reduced heparan sulfate accumulation in enterocytes contributes to protein-losing enteropathy in a congenital disorder of glycosylation. Am J Pathol 157:1917-1925

12. Packer NH, Lawson MA, Jardine DR, Redmond JW 1998 A general approach to desalting oligosaccharides released from glycoproteins. Glycoconj J 15:737-747

13. Bigge JC, Patel TP, Bruce JA, Goulding PN, Charles SM, Parekh RB 1995 Nonselective and efficient fluorescent labeling of glycans using 2-amino benzamide and anthranilic acid. Anal Biochem 230:229-238

14. Townsend RR, Lipniunas PH, Bigge C, Ventom A, Parekh R 1996 Multimode high-performance liquid chromatography of fluorescently labeled oligosaccharides from glycoproteins. Anal Biochem 239:200-207

15. Kuster B, Hunter AP, Wheeler SF, Dwek RA, Harvey DJ 1998 Structural determination of $\mathrm{N}$-linked carbohydrates by matrix-assisted laser desorption/ionization-mass spectrometry following enzymatic release within sodium dodecyl sulphatepolyacrylamide electrophoresis gels: application to species-specific glycosylation of alpha1-acid glycoprotein. Electrophoresis 19:1950-1959 
16. Papac DI, Briggs JB, Chin ET, Jones AJ 1998 A high-throughput microscale method to release $\mathrm{N}$-linked oligosaccharides from glycoproteins for matrix-assisted laser desorption/ionization time-of-flight mass spectrometric analysis. Glycobiology $8: 445-454$

17. de Lonlay P, Seta N, Barrot S, Chabrol B, Drouin V, Gabriel BM, Journel H, Kretz M, Laurent J, Le Merrer M, Leroy A, Pedespan D, Sarda P, Villeneuve N, Schmitz J, van Schaftingen E, Matthijs G, Jaeken J, Korner C, Munnich A, Saudubray JM, Cormier-Daire V 2001 A broad spectrum of clinical presentations in congenital disorders of glycosylation I: a series of 26 cases. J Med Genet 38:14-19

18. Cheillan D, Cognat S, Dorche C, Jaeken J, Vianey-Saban C, Guffon N 2004 CDG IIx with unusual phenotype. J Inherit Metab Dis 27:103-104

19. Jaeken J, Carchon H 2004 Congenital disorders of glycosylation: a booming chapter of pediatrics. Curr Opin Pediatr 16:434-439

20. Morava E, Wopereis S, Coucke P, Gillessen-Kaesbach G, Voit T, Smeitink J, Wevers R, Grunewald S 2005 Defective protein glycosylation in patients with cutis laxa syndrome. Eur J Hum Genet 13:414-421

21. Jaeken J 2004 Congenital disorders of glycosylation (CDG): update and new developments. J Inherit Metab Dis 27:423-426

22. Eyskens F, Ceuterick C, Martin JJ, Janssens G, Jaeken J 1994 Carbohydratedeficient glycoprotein syndrome with previously unreported features. Acta Paediatr 83:892-896

23. Acarregui MJ, George TN, Rhead WJ 1998 Carbohydrate-deficient glycoprotein syndrome type 1 with profound thrombocytopenia and normal phosphomannomutase and phosphomannose isomerase activities. J Pediatr 133:697-700

24. Charlwood J, Clayton P, Johnson A, Keir G, Mian N, Winchester B 1997 A case of the carbohydrate-deficient glycoprotein syndrome type 1 (CDGS type 1) with normal phosphomannomutase activity. J Inherit Metab Dis 20:817-826

25. Skladal D, Sperl W, Henry H, Bachmann C 1996 Congenital cataract and familial brachydactyly in carbohydrate-deficient glycoprotein syndrome. J Inherit Metab Dis 19:251-252
26. Damen G, de Klerk H, Huijmans J, den Hollander, J, Sinaasappel M 2004 Gastrointestinal and other clinical manifestations in 17 children with congenital disorders of glycosylation type Ia, Ib, and Ic. J Pediatr Gastroenterol Nutr 38:282-287

27. Barone R, Pavone V, Pennisi P, Fiumara A, Fiore CE 2002 Assessment of skeleta status in patients with congenital disorder of glycosylation type IA. Int J Tissue React 24:23-28

28. Ferens-Sieczkowska M, Zwierz K, Midro A, Katnik-Prastowska I Glycoforms of six serum glycoproteins in a patient with congenital disorder of glycosylation type I. Arch Immunol Ther Exp (Warsz) 2002;50:67-73

29. Ferens-Sieczkowska M, Midro A, Mierzejewska-Iwanowska B, Zwierz K, KatnikPrastowska I 1999 Haptoglobin glycoforms in a case of carbohydrate-deficient glycoprotein syndrome. Glycoconj J 16:573-577

30. Stibler H, Holzbach U, Kristiansson B 1998 Isoforms and levels of transferrin, antithrombin, alpha(1)-antitrypsin and thyroxine-binding globulin in 48 patients with carbohydrate-deficient glycoprotein syndrome type I. Scand J Clin Lab Invest 58:55-61

31. Ferenci P 2004 Review article: diagnosis and current therapy of Wilson's disease. Aliment Pharmacol Ther 15; 19:157-165

32. Callewaert N, Schollen E, Vanhecke A, Jaeken J, Matthijs G, Contreras R 2003 Increased fucosylation and reduced branching of serum glycoprotein N-glycans in all known subtypes of congenital disorder of glycosylation I. Glycobiology $13: 367-675$

33. Callewaert N, Van Vlierberghe, H, Van Hecke, A, Laroy W, Delanghe J, Contreras R 2004 Noninvasive diagnosis of liver cirrhosis using DNA sequencer-based total serum protein glycomics. Nat Med 10:429-434

34. Wopereis S, Grunewald S, Morava E, Penzien JM, Briones P, Garcia-Silva MT, Demacker PN, Huijben KM, Wevers RA 2003 Apolipoprotein C-III isofocusing in the diagnosis of genetic defects in O-glycan biosynthesis. Clin Chem 49:1839-1845 